\title{
Activating PIK3CA Gene Mutation
}

National Cancer Institute

\section{Source}

National Cancer Institute. Activating PIK3CA Gene Mutation. NCI Thesaurus. Code C142116.

A change in the nucleotide sequence of the PIK3CA gene that that results in constitutive activation of phosphatidylinositol 4,5-bisphosphate 3-kinase catalytic subunit alpha isoform and its downstream signaling pathways. 